Pacific

Journal of

Mathematics

\title{
REDUCIBLE DEHN SURGERY AND ANNULAR DEHN SURGERY
}

RUIFENG QIU 


\title{
REDUCIBLE DEHN SURGERY AND ANNULAR DEHN SURGERY
}

\author{
RUIFEng QIU
}

\begin{abstract}
Let $M$ be a compact, orientable, irreducible, $\partial$-irreducible, anannular 3-manifold with one component $T$ of $\partial M$ a torus. Suppose that $r_{1}$ and $r_{2}$ are two slopes on $T$. In this paper, we shall show that if $M\left(r_{1}\right)$ is reducible while $M\left(r_{2}\right)$ contains an essential annulus, then $\triangle\left(r_{1}, r_{2}\right) \leq 2$.
\end{abstract}

\section{Introduction.}

Let $M$ be a compact, orientable, irreducible, $\partial$-irreducible, anannular 3manifold with one component $T$ of $\partial M$ a torus. A slope $r$ on $T$ is a $T$ isotopy class of essential, unoriented, simple closed curves on $T$, and the distance between two slopes $r_{1}$ and $r_{2}$, denoted by $\triangle\left(r_{1}, r_{2}\right)$, is the minimal geometric intersection number among all the curves representing the slopes. For a slope $r$ on $T$, we denote by $M(r)$ the surgered manifold obtained by attaching a solid torus $J$ to $M$ along $T$ so that $r$ bounds a disk in $J$. Now consider two distinct slopes $r_{1}, r_{2}$ on $T$. There are many results showing how constraints on the topology of $M\left(r_{1}\right)$ and $M\left(r_{2}\right)$ put constraints on $\triangle\left(r_{1}, r_{2}\right)$. For example, C. Gordon and J. Luecke [5] have shown that if $M\left(r_{1}\right)$ and $M\left(r_{2}\right)$ are reducible, then $\triangle\left(r_{1}, r_{2}\right) \leq 1$. C. Gordon [3] has shown that if $M$ contains no essential torus, and $M\left(r_{i}\right)$ contains an essential torus, $i=1,2$, then $\triangle\left(r_{1}, r_{2}\right) \leq 8$. Y-Q Wu [8] has shown that if $M\left(r_{1}\right)$ and $M\left(r_{2}\right)$ are $\partial$-reducible, then $\triangle\left(r_{1}, r_{2}\right) \leq 1$. In this paper, we shall estimate $\triangle\left(r_{1}, r_{2}\right)$ when $M\left(r_{1}\right)$ is reducible, and $M\left(r_{2}\right)$ contains an essential annulus. The main result is the following theorem:

Theorem 1. Let $M$ be a compact, orientable, irreducible, $\partial$-irreducible, anannular 3-manifold with one component $T$ of $\partial M$ a torus. If $r_{1}$ and $r_{2}$ are two slopes on $T$, such that $M\left(r_{1}\right)$ is reducible while $M\left(r_{2}\right)$ contains an essential annulus, then $\triangle\left(r_{1}, r_{2}\right) \leq 2$.

An example has been given by Hayashi and Motegi [6] showing that the bound 2 in Theorem 1 is the best possible in general.

Another proof of Theorem 1 has been obtained independently by Y-Q $\mathrm{Wu}$. 


\section{Scharlemann cycle and parallel edges.}

In what follows, we shall assume that $M\left(r_{1}\right)$ is reducible, and $M\left(r_{2}\right)$ contains an essential annulus. We may assume further that $M\left(r_{2}\right)$ is irreducible, $\partial$ irreducible (see [5], [7]).

Let $V_{i}$ be the solid torus attached to $M$ in forming $M\left(r_{i}\right), i=1,2$. Consider the family of essential 2-spheres in $M\left(r_{1}\right)$ which intersect $V_{1}$ in a family of meridional discs, and let $S \subset M\left(r_{1}\right)$ be such a 2-sphere chosen so that $S \cap V_{1}$ has the minimal number, say $n_{1}$, of components. Similarly, let $A \subset M\left(r_{2}\right)$ be an essential annulus which intersects $V_{2}$ in a collection of meridian discs, the number of which, say $n_{2}$, is minimal among all such annuli. By assumptions, $n_{1}>2$ and $n_{2}>0$.

Now suppose that $F_{1}=M \cap S$ and $F_{2}=M \cap A$. Then $F_{1}$ is an essential planar surface in $M$ with boundary slope $r_{1}$ while $F_{2}$ is an essential punctured annulus in $M$ with boundary slope $r_{2}$. We may assume that the number of components of $F_{1} \cap F_{2}$ is minimal subject to these conditions. Then no circle component of $F_{1} \cap F_{2}$ bounds a disk in $F_{1}$ or $F_{2}$, and no arc component of $F_{1} \cap F_{2}$ is boundary parallel in $F_{1}$ or $F_{2}$. Each component of $\partial F_{i}$ lying in $T$ is called an inner component of $\partial F_{i}, i=1,2$.

Let $\Gamma_{1}\left(\Gamma_{2}\right)$ be the graph in $S(A)$ obtained by taking the arc components of $F_{1} \cap F_{2}$ as edges and taking the inner components of $\partial F_{1}\left(\partial F_{2}\right)$ as fat vertices.

We shall use the indices $\alpha$ and $\beta$ to denote 1 or 2 , with the convention that, when they are used together, $\{\alpha, \beta\}=\{1,2\}$.

Number the inner components of $\partial F_{\alpha}, \partial_{1} F_{\alpha}, \ldots, \partial_{n_{\alpha}} F_{\alpha}$, so that they appear consecutively on $T$. By construction, each inner component $\partial_{i} F_{\alpha}$ of $\partial F_{\alpha}$ intersects each inner component $\partial_{j} F_{\beta}$ of $\partial F_{\beta}$ in exactly $\Delta\left(r_{1}, r_{2}\right)$ points. The ends of the edges in $\Gamma_{\alpha}$ may be labeled by an integer $k \in\left\{1,2, \ldots, n_{\beta}\right\}$ as follows. Let $x$ be the intersection of an edge $e$ of $\Gamma_{\alpha}$ with one of its vertices $\partial_{i} F_{\alpha}$, then $x$ is labeled $k$, where $\partial_{k} F_{\beta}$ is the unique vertex of $\Gamma_{\beta}$, such that $x \in e \cap \partial_{i} F_{\alpha} \cap \partial_{k} F_{\beta}$. Thus when we travel around $\partial_{i} F_{\alpha}$, the labels appear in the order $1, \ldots, n_{\beta}, \ldots, 1, \ldots, n_{\beta}$ (repeated $\triangle\left(r_{1}, r_{2}\right)$ times).

Now fix an orientation on $F_{\alpha}$, and let each inner component $\partial_{i} F_{\alpha}$ of $\partial F_{\alpha}$ have the induced orientation. Two inner components of $\partial F_{\alpha}$ are said to be parallel if they, when given the induced orientation by $F_{\alpha}$, are homologous on $T$; otherwise they are antiparallel. Two vertices of $\Gamma_{\alpha}$ are said to be parallel if the corresponding inner components of $\partial F_{\alpha}$ are parallel; otherwise they are antiparallel.

Parity rule [2]. An edge connects parallel vertices of $\Gamma_{\alpha}$ if and only if it connects antiparallel vertices of $\Gamma_{\beta}$.

Two edges of $\Gamma_{\alpha}$ are said to be parallel if they, together with some arcs in $\partial F_{\alpha}$, bound a disk in $F_{\alpha}$. A cycle $\sigma$ in $\Gamma_{\alpha}$ is a subgraph of $\Gamma_{\alpha}$ homeomorphic to a circle. The length of a cycle is the number of edges contained in it. A 
cycle $\sigma$ in $\Gamma_{\alpha}$ is called a Scharlemann cycle if it bounds a disk face of $\Gamma_{\alpha}$ and the edges of $\sigma$ connect parallel vertices of $\Gamma_{\alpha}$, and have the same two labels at their ends. A length two Scharlemann cycle is called an S-cycle. A length two cycle $\sigma^{\prime}=\left\{e_{1}^{\prime}, e_{2}^{\prime}\right\}$ in $\Gamma_{\alpha}$ is called an extended $S$-cycle if there is an $S$-cycle $\sigma=\left\{e_{1}, e_{2}\right\}$ in $\Gamma_{\alpha}$ such that $e_{i}^{\prime}$ and $e_{i}$ are adjacent parallel edges in $\Gamma_{\alpha}, i=1,2$.

Let $x$ be a vertex of $\Gamma_{\alpha}$. An edge of $\Gamma_{\beta}$ is called an $x$-edge if it has label $x$ at one of its two ends. We denote by $\Gamma_{\beta}^{x}$ the subgraph of $\Gamma_{\beta}$ consisting of all the vertices of $\Gamma_{\beta}$ and the $x$-edges connecting parallel vertices of $\Gamma_{\beta}$. A disk face $D$ of $\Gamma_{\beta}^{x}$ is called an $x$-face.

Lemma 2.1. Let $W$ be a compact, irreducible, $\partial$-irreducible 3-manifold, and let $A_{0}$ be a non-separating annulus properly embedded in $W$. Then $A_{0}$ is essential in $W$.

Proof. Let $D$ be a compressing disk of $A_{0}$. If one component of $\partial A_{0}$ is essential on $\partial W$, then $W$ is $\partial$-reducible, a contradiction. If the two components of $\partial A_{0}$ are trivial on $\partial W$, then $W$ contains a non-separating 2-sphere. Thus $W$ is reducible, a contradiction.

Now let $D$ be a $\partial$-compressing disk of $A_{0}$, such that $\partial D=a \cup b$, where $a$ is an $\operatorname{arc}$ on $A_{0}$, and $b$ is an arc on $\partial W$. If the two components of $\partial A_{0}$ bounds an annulus $A_{1}$ on $\partial W$, and $b \subset A_{1}$, then $A_{0} \cup A_{1}$ is non-separating in $W$, and the surface obtained by doing a 2-surgery on $A_{0} \cup A_{1}$ along $D$ is a non-separating 2 -sphere in $W$. Thus $W$ is reducible, a contradiction. If not, then the band connected sum of the two components of $\partial A_{0}$ along $b$ on $\partial W$, say $C$, bounds a disk in $W$, and $C$ is essential on $\partial W$. Thus $W$ is $\partial$-reducible, a contradiction.

Lemma 2.2. If $\Gamma_{\alpha}$ contains a Scharlemann cycle, then $F_{\beta}$ is separating.

Proof. By Lemma 2.1 of [5], $F_{1}$ is separating when $\Gamma_{2}$ contains a Scharlemann cycle.

Now let $\sigma$ be a Scharlemann cycle of $\Gamma_{1}$ with label pair $\{1,2\}, D$ be the disk face bounded by $\sigma$ in $\Gamma_{1}$, and let $A_{1}$ be the annulus bounded by $\partial_{1} F_{2}$ and $\partial_{2} F_{2}$ on $T$, such that the interior of $A_{1}$ is disjoint from $A$. Let $D_{i}$ be the disks in $A$ bounded by $\partial_{i} F_{2}$, and let $T^{\prime}=\left(A-D_{1} \cup D_{2}\right) \cup A_{1}$. Then $T^{\prime}$ is a punctured torus. Let $A^{\prime}$ be the surface obtained by doing a 2 -surgery on $T^{\prime}$ along $D$, then $A^{\prime}$ is an annulus in $M\left(r_{2}\right)$, such that $\left|A^{\prime} \cap V_{2}\right|<n_{2}$. If $F_{2}$ is non-separating, then $A^{\prime}$ is also non-separating. By Lemma 2.1, $A^{\prime}$ is essential, contradicting the minimality of $n_{2}$.

Lemma 2.3. Let $\sigma$ be a Scharlemann cycle of $\Gamma_{1}$, then the edges in $\sigma$ can not lie in a disk of $A$.

Proof. Suppose, otherwise, that the edges in $\sigma$ lie in a disk of $A$. Then $M\left(r_{2}\right)$ contains a lens space as a factor (by the proof of Lemma 2.8 of [1]). 
Since $\partial M\left(r_{2}\right) \neq \phi, M\left(r_{2}\right)$ is reducible, contradicting our assumptions on $M\left(r_{2}\right)$.

Proposition 2.4. $\Gamma_{\alpha}$ can not contain two Scharlemann cycles with distinct label pairs.

Proof. By Theorem 2.4 of [5], $\Gamma_{2}$ can not contain two Scharlemann cycles with distinct label pairs.

Now suppose, otherwise, that $\Gamma_{1}$ contains two Scharlemann cycles $\sigma_{1}$ and $\sigma_{2}$, with label pairs $\{x, y\}$ and $\left\{x^{\prime}, y^{\prime}\right\}$ respectively, such that $\{x, y\} \neq$ $\left\{x^{\prime}, y^{\prime}\right\}$. By Lemma 2.2, $n_{2}$ is even.

Now consider the edges of $\sigma_{1}$ and $\sigma_{2}$ as they lie in $\Gamma_{2}$, joining the vertices $x, y$ and $x^{\prime}, y^{\prime}$. By Lemma 2.3, there exists an annulus $E \subset A$, such that

1) one component of $\partial E$ is one component of $\partial A$, say $\partial_{1} A$, and another component of $\partial E$ is contained in $\operatorname{int} A$;

2) the number of vertices of $\Gamma_{2}$ in $E$ is at most $n_{2} / 2$;

$3)$ int $E$ contains the edges of one of the two Scharlemann cycles, say $\sigma_{1}$, and the corresponding vertices $x, y$.

Let $E^{\prime}$ be an annulus containing $x^{\prime}, y^{\prime}$ and the edges of $\sigma_{2}$, such that one component of $\partial E^{\prime}$ is the remaining component of $\partial A$, say $\partial_{2} A$, and another component of $\partial E^{\prime}$ is contained in $\operatorname{int} A$. If $\{x, y\} \cap\left\{x^{\prime}, y^{\prime}\right\}=\phi$, then we may assume $E \cap E^{\prime}=\phi$.

Now let $D$ be the face of $\Gamma_{1}$ bounded by $\sigma_{1}$, let $H$ be the 3-cell in $V_{2}$ between the consecutive meridional disks of $V_{2}$ corresponding to $x$ and $y$, and let $N$ be a regular neighborhood of $E \cup H \cup D$ in $M\left(r_{2}\right)$. Then the frontier of $N$ is an annulus $A^{\prime}$ properly embedded in $M\left(r_{2}\right)$, whose two boundary components are $\partial_{1} A \times\{-1\}$ and $\partial_{1} A \times\{1\}$, and the union of $N$ and $D_{0} \times[-1,1]$ along $\partial_{1} A \times[-1,1]$ is a punctured lens space whose fundamental group has order the length of $\sigma_{1}$, where $D_{0}$ is a disk. Similarly, let $D^{\prime}$ be the face of $\Gamma_{1}$ bounded by $\sigma_{2}$, let $H^{\prime}$ be the 3 -cell in $V_{2}$ between the consecutive meridional disks of $V_{2}$ corresponding to $x^{\prime}, y^{\prime}$, and let $N^{\prime}$ be a regular neighborhood of $E^{\prime} \cup H^{\prime} \cup D^{\prime}$. Then the frontier of $N^{\prime}$ is an annulus $A^{\prime \prime}$ properly embedded in $M\left(r_{2}\right)$, whose two boundary components are $\partial_{2} A \times\{-1\}$ and $\partial_{2} A \times\{1\}$, and the union of $N^{\prime}$ and $D_{0} \times[-1,1]$ along $\partial_{2} A \times[-1,1]$, say $M_{1}$, is a punctured lens space whose fundamental group has order the length of $\sigma_{2}$, where $D_{0}$ is a disk. We may assume that $N \cap N^{\prime}=\phi$ (moving $\partial N$ slightly off $A$ if $\{x, y\} \cap\left\{x^{\prime}, y^{\prime}\right\} \neq \phi$ ). We claim that $A^{\prime}$ is essential in $M\left(r_{2}\right)$.

Suppose, otherwise, that $A^{\prime}$ is not essential in $M\left(r_{2}\right)$. Since $M\left(r_{2}\right)$ is $\partial$ irreducible, $A^{\prime}$ is incompressible in $M\left(r_{2}\right)$. Now let $D_{1}$ be a $\partial$-compressing disk of $A^{\prime}$, such that $\partial D_{1}=a \cup b, a \subset \partial M\left(r_{2}\right)$, and $b \subset A^{\prime}$.

Case 1. $a \subset \partial_{1} A \times[-1,1]$. 
Now either $M\left(r_{2}\right)$ is reducible, or the union of $N$ and $D_{0} \times[-1,1]$ along $\partial_{1} A \times[-1,1]$ is a 3 -cell, a contradiction.

Case 2. $a \subset \partial M\left(r_{2}\right)-\partial_{1} A \times(-1,1)$.

If $\partial M-\partial_{1} A \times(-1,1)$ is not an annulus, then either $M\left(r_{2}\right)$ is reducible, or $M\left(r_{2}\right)$ is $\partial$-reducible, a contradiction. If $\partial M-\partial_{1} A \times(-1,1)$ is an annulus, then $\partial M\left(r_{2}\right)$ is a torus, and the union of $M\left(r_{2}\right)-\operatorname{int} N$ and $D_{0} \times[-1,1]$ along $\partial M-\partial_{1} A \times(-1,1)$ is a 3 -cell, but it contains $M_{1}$ as a factor, a contradiction.

By construction, $\left|A^{\prime} \cap V_{2}\right|=\left|A \cap V_{2}\right|-2$, contradicting the minimality of $n_{2}$.

Lemma 2.5. (1) $\Gamma_{2}$ contains no extended $S$-cycle.

(2) $\Gamma_{2}$ contains at most $n_{1} / 2+1$ mutually parallel edges connecting parallel vertices.

(3) $\Gamma_{2}$ contains at most $n_{1}-1$ mutually parallel edges.

(4) If $\Gamma_{1}$ contains a great cycle, then $\Gamma_{1}$ contains a Scharlemann cycle.

(5) If $n_{\alpha} \geq 3$, and $\Gamma_{\beta}$ contains two distinct Scharlemann cycles $\sigma_{1}$ and $\sigma_{2}$, then the edges of $\sigma_{1}$ are disjoint from the edges of $\sigma_{2}$.

Proof. (1) is Lemma 2.3 of [9]. (2) is Lemma 2.4 of [9]. (3) is Lemma 2.6 of [1]. See also [4, Proposition 1.3]. (4) is Lemma 2.6.2 of [2]. (5) Suppose, otherwise, that one edge of $\sigma_{1}$ is contained in $\sigma_{2}$. Then $n_{\alpha}=2$, a contradiction.

Lemma 2.6. Let $y$ be a vertex of $\Gamma_{\beta}$.

(1) If $\Gamma_{\alpha}$ contains a n-sided $y$-face, such that $2 \leq n \leq 3$, then $\Gamma_{\alpha}$ contains a Scharlemann cycle.

(2) If $F_{\beta}$ is separating, and $\Gamma_{\alpha}$ contains a $y$-face $f$, then $\Gamma_{\alpha}$ contains a Scharlemann cycle in $f$.

Proof. (1) Suppose that $\Gamma_{\alpha}$ contains a $n$-sided $y$-face, such that $2 \leq n \leq 3$. Then $\Gamma_{\alpha}$ contains a great cycle. By Lemma 2.5(4), $\Gamma_{\alpha}$ contains a Scharlemann cycle. (2) is Lemma 2.2 of [5].

\section{Reduced graph.}

Let $G$ be a graph in a surface $S$. The reduced graph of $G$ is the graph obtained from $G$ by amalgamating each complete set of mutually parallel edges of $G$ to a single edge.

Lemma 3.1. One of $\Gamma_{1}$ and $\Gamma_{2}$ satisfies

(*). Each vertex is incident to an edge connecting it to an antiparallel vertex.

This follows immediately from the proof of [9, Lemma 2.6]. 
Let $G_{\alpha}$ be the subgraph of $\Gamma_{\alpha}$ consisting of all the vertices of $\Gamma_{\alpha}$ and the edges connecting parallel vertices. We first suppose that $\Gamma_{2}$ has property (*). A component $F^{\prime}$ of $G_{2}$ is called an extremal component if there is a disc $D$ in $\widehat{A}$ such that $D \cap G_{2}=F^{\prime}$, where $\widehat{A}$ is the 2 -sphere obtained by capping off the two components of $\partial A$ with disks. In this case $F=D \cap \Gamma_{2}$ is a graph in $D$. If $e$ is an edge in $\Gamma_{2}$ connecting a vertex of $F^{\prime}$ to an antiparallel vertex, then $e \cap D$ is an edge of $F$ connecting that vertex to $\partial D$. Such an edge is called a boundary edge of $F$. Property $(*)$ means that each vertex of $F$ belongs to a boundary edge.

Lemma 3.2. Let $\Gamma$ be a graph in a disk with no 1-sided disk face or two sided disk face, such that every vertex of $\Gamma$ belongs to a boundary edge, then either $\Gamma$ contains only one vertex, or there are at least two vertices of valency at most 3, each of which belongs to a single boundary edge.

This follows immediately from the proof of [2, Lemma 2.6.5].

Lemma 3.3. If $\Gamma_{2}$ has property $(*)$, then there exists at least one vertex of $\Gamma_{2}$, such that among the families of ends around it, there are at most two families which are ends of edges connecting it to parallel vertices. Furthermore, if there are two such families, they are successive.

Proof. Since $G_{2}$ contains at least two extremal components, there is an extremal component $F^{\prime}$ of $G_{2}$, such that the correspond disc $D$ of $F^{\prime}$ in $\widehat{A}$ contains at most one component of $\partial A$, say $\partial_{1} A$, and the remaining component of $\partial A$ is disjoint from $D$. Thus $F$ contains at most one 1 -sided disk face. Furthermore, if $F$ contains a 1 -sided disk face $f_{1}$, then $\partial_{1} A \subset \operatorname{int} f_{1}$. Let $\bar{F}$ be the reduced graph of $F$ in $D$. Let $S$ be the graph obtained from $\bar{F}$ by removing the edge bounding $f_{1}$. (Let $S=\bar{F}$ when $\bar{F}$ contains no 1-sided disk face.) Then $S$ contains no 1-sided disk face, and it contains at most one 2-sided disk face. Furthermore, if $S$ contains a 2 -sided disk face $f_{2}$, then $\partial_{1} A\left(\subset \operatorname{int} f_{1}\right) \subset \operatorname{int} f_{2}$. Let $\bar{S}$ be the reduced graph of $S$ in $D$. Let $D_{0}$ be the disk bounded by $\partial_{1} A$ in $D$, and let $\bar{F}_{0}$ be the reduced graph of $F$ in $D$-int $D_{0}$.

To prove the lemma, we need only to prove that $\bar{F}_{0}$ contains a vertex of valency at most 3 , which belongs to a boundary edge. If $F^{\prime}$ contains only one vertex $v$, then $v$ has valency 3 in $\bar{F}_{0}$, and it belongs to a boundary edge. If $F^{\prime}$ contains two vertices, then the one which does not belong to the edge bounding $f_{1}$, has valency at most 3 in $\bar{F}_{0}$. Assume now that $F^{\prime}$ contains at least three vertices. 


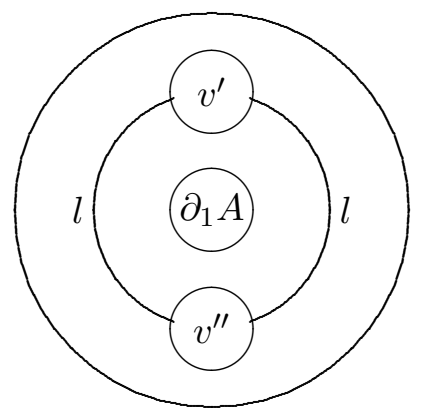

Figure 1.

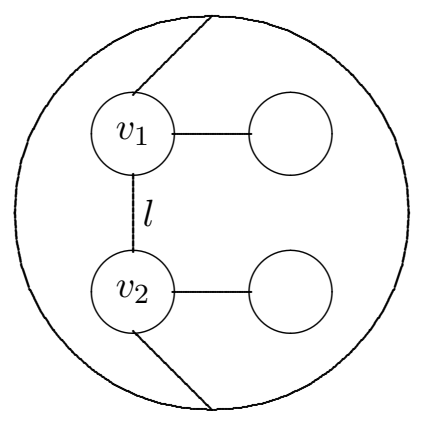

Figure 2.

Case 1. $S$ contains no 2 -sided disk face.

By Lemma 3.2, $S$ contains at least two vertices of valency at most 3, say $v_{1}$ and $v_{2}$, each of which belongs to a single boundary edge.

Suppose that $\partial_{1} A$ is contained in a fat edge $l$ of $S$ (as in Fig. 1). If $v_{i} \notin\left\{v^{\prime}, v^{\prime \prime}\right\}, i=1$, or 2 , then $v_{i}$ has valency at most 3 in $\bar{F}_{0}$. If $\left\{v_{1}, v_{2}\right\}=$ $\left\{v^{\prime}, v^{\prime \prime}\right\}$, then the edges incident to one of $v_{1}$ and $v_{2}$ are as in one of Figures $2-4$.

(1) the edges incident to one of $v_{1}$ and $v_{2}$ are as in Fig. 2.

Let $S^{\prime}$ be the graph obtained from $S$ by taking the union of $v_{1}, l$ and $v_{2}$ as a fat vertex, say $v$. Then $S^{\prime}$ contains no 1 -sided disk face or 2 -sided disk face. By Lemma 3.2, $S^{\prime}$ contains at least two vertices of valency at most 3 , each of which belongs to a single boundary edge, and the one which is not equal to $v$, has valency at most 3 in $\bar{F}_{0}$. 


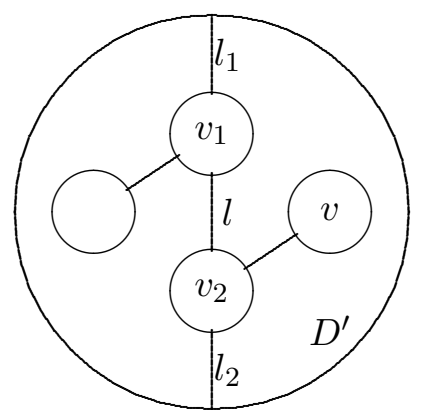

Figure 3.

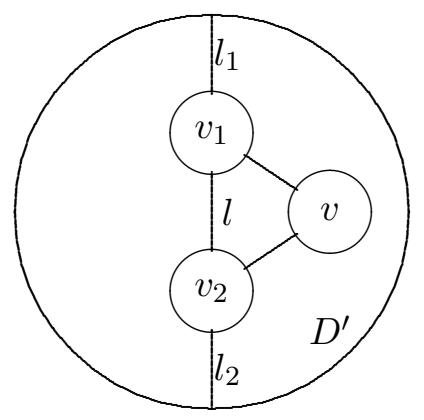

Figure 4.

(2) the edges incident to one of $v_{1}$ and $v_{2}$ are as in one of Figures 3-4.

Now $l_{1} \cup v_{1} \cup l \cup v_{2} \cup l_{2}$ separates $D$ into two discs $D^{\prime}$ and $D^{\prime \prime}$. Since $\partial_{1} A$ is contained in $l$, the graph $S \cap D^{\prime}$ contains no 1-sided disk face or 2-sided disk face. If $S \cap D^{\prime}$ contains only one vertex $v$, then $v$ has valency at most 3 in $\bar{F}_{0}$. If $S \cap D^{\prime}$ contains at least two vertices, then $S \cap D^{\prime}$ contains at least two vertices of valency at most 3 , each of which belongs to a single boundary edge, and the one which is not equal to $v$, has valency at most 3 in $\bar{F}_{0}$.

Now we suppose that $\partial_{1} A$ is not contained in a fat edge. Since $S$ contains no 2-sided disk face, the one of $v_{1}$ and $v_{2}$ which does not belong to the edge bounding $f_{1}$, has valency at most 3 in $\bar{F}_{0}$.

Case 2. $S$ contains a 2 -sided disk face $f_{2}$.

Now $\partial_{1} A \subset \operatorname{int} f_{2}$. That means that $\partial_{1} A$ is contained in a fat edge of $\bar{S}$. By Lemma 3.2, $\bar{S}$ contains at least two vertices of valency at most 3 , each 
of which belongs to a single edge. Using $\bar{S}$ to take place of $S$ in Case 1, we can proof that $\bar{F}_{0}$ contains a vertex of valency at most 3 , which belongs to a boundary edge.

Proposition 3.4. If $\Gamma_{2}$ has property $(*)$, then $\Gamma_{2}$ contains at least one vertex, such that around it, all the endpoints of edges connecting it to parallel vertices are successive, and there are at most $n_{1}+2$ of them.

Proof. This follows immediately from Lemma 2.5(2) and Lemma 3.3.

Proposition 3.5. If $\Gamma_{2}$ does not have property $(*)$, then $\Gamma_{1}$ contains at least one vertex, such that around it, all the endpoints of edges connecting to parallel vertices are successive, and there are at most $n_{2}-1$ of them.

Proof. By Lemma 3.1, $\Gamma_{1}$ has property $(*)$. Now let $F^{\prime}$ be an extremal component of $G_{1}$, and let $D$ be the corresponding disc. In this case $F=$ $D \cap \Gamma_{1}$ is a graph in $D$. By Lemma $3.2, \bar{F}$ contains at least one vertex, say $x$, of valency at most 3 , which belongs to a single boundary edge. That implies that in $\Gamma_{1}$, there are at most two families of parallel edges connecting $x$ to parallel vertices, and if there are two such families, then they are successive. Since $\Gamma_{2}$ does not have property $(*), \Gamma_{2}$ contains one vertex, such that each of the edges incident to it connects it to a parallel vertex. That implies that all edges in $\Gamma_{1}$ with this vertex as a label connect nonparallel vertices, hence the above two families of parallel edges contains at most $n_{2}-1$ edges.

\section{The proof of Theorem 1 .}

Let $G$ be a graph on a surface $S$. In this section, we shall denote by $V$ the number of vertices of $G, E$ the number of edges of $G$ and $F$ the number of disk faces of $G$. By the Euler characteristic formula, $V-E+F \geq \chi(S)$.

Proposition 4.1. If $n_{2}=1$, then $\triangle\left(r_{1}, r_{2}\right) \leq 1$.

Proof. Suppose, otherwise, that $\triangle\left(r_{1}, r_{2}\right) \geq 2$. Since $n_{2}=1, \Gamma_{2}$ contains only one vertex, say $x$. It is easy to see that $x$ has valency 2 in $\bar{\Gamma}_{2}$. Hence $\Gamma_{2}$ contains $n_{1}$ mutually parallel edges, contradicting Lemma 2.5(3).

Proposition 4.2. If $n_{2}=2$, then $\triangle\left(r_{1}, r_{2}\right) \leq 2$.

Proof. Suppose, otherwise, that $\triangle\left(r_{1}, r_{2}\right) \geq 3$. Since $n_{2}=2$, each vertex of $\bar{\Gamma}_{2}$ has valency 4 as in Fig. 5 (otherwise $\Gamma_{2}$ contains $n_{1}$ mutually parallel edges). By Lemma 2.5(2), $l_{1}$ and $l_{2}$ contains at most $n_{1}+2$ edges. If $l_{1}$ and $l_{2}$ occupy at most $n_{1}+1$ edges, then one of $l_{3}$ and $l_{4}$ occupies at least $n_{1}$ mutually parallel edges, contradicting Lemma 2.5(3). 


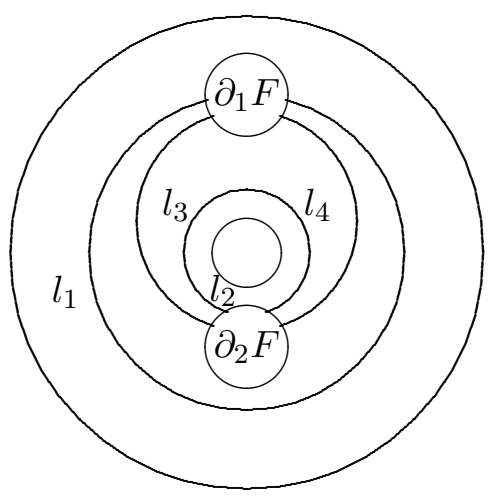

Figure 5.

Now we suppose that that $l_{1}$ and $l_{2}$ occupy $n_{1}+2$ edges. Then $\Gamma_{2}$ contains an S-cycle. By Lemma 2.2, $F_{1}$ is separating, and $n_{1} \geq 4$. By Lemma 2.5(3), each of $l_{3}$ and $l_{4}$ occupies $n_{1}-1$ edges.

Case 1. $\partial_{1} F_{2}$ and $\partial_{2} F_{2}$ are parallel.

Let $x$ be a vertex of $\Gamma_{1}$. By the parity rule, $\Gamma_{2}^{x}$ contains at least six $\left(3 n_{2}\right)$ edges. Since $\bar{\Gamma}_{2}$ has four edges, $\Gamma_{2}^{x}$ contains a 2 -sided disk face. By Lemma 2.5(1) and Lemma 2.5(3), $\Gamma_{2}$ contains an S-cycle, one label of which is $x$, for any given vertex $x$ in $\Gamma_{1}$. Since $n_{1} \geq 3, \Gamma_{2}$ contains two Scharlemann cycles with distinct label pairs, contradicting Proposition 2.4.

Case 2. $\partial_{1} F_{2}$ and $\partial_{2} F_{2}$ are antiparallel.

Let $\Gamma$ be the subgraph of $\Gamma_{1}$ consisting of all the vertices of $\Gamma_{1}$ and the edges in $l_{3}$. Since $F_{1}$ is separating, $\Gamma$ is not connected. By the Euler characteristic formula, $\Gamma$ contains a disk face. By the proof of Proposition 1.3 of [4], $M$ contains an essential annulus, a contradiction.

In the following arguments, we shall assume that $n_{\alpha} \geq 3$.

Proposition 4.3. If $\Gamma_{2}$ has property $(*)$, then $\triangle\left(r_{1}, r_{2}\right) \leq 2$.

Proof. Suppose, otherwise, that $\triangle\left(r_{1}, r_{2}\right) \geq 3$. By Proposition 3.4, there exists a vertex of $\Gamma_{2}$, say $y$, such that $\Gamma_{1}^{y}$ contains at least $2 n_{1}+l$ edges, where $l \geq-2$. By the Euler characteristic formula, $\Gamma_{1}^{y}$ contains at least $n_{1}+l+2 \geq n_{1}$ disk faces. Since there are $n_{1}$ adjacent edges at $y$ connecting it to antiparallel vertices, there is a great $y$-cycle in $\Gamma_{1}$. By Lemma $2.5(4), \Gamma_{1}$ contains a Scharlemann cycle. By Lemma $2.2, F_{2}$ is separating. By Lemma 2.6(2) and Proposition 2.4, $\Gamma_{1}$ contains at least $n_{1}$ Scharlemann cycles with the same label pair, say $\{1,2\}$. Now suppose that $\Gamma_{1}$ contains $m$ Scharlemann cycles with label pair $\{1,2\}$. Then $m \geq n_{1}$. By Lemma 2.5(5), $\Gamma_{1}^{1}$ contains at least $2 m$ edges. By the Euler characteristic formula, $\Gamma_{1}^{1}$ contains 
at least $2 m-n_{1}+2 \geq m+2$ disk faces. By Lemma 2.6(2), $\Gamma_{1}$ contains at least $m+2$ Scharlemann cycles. Thus $\Gamma_{1}$ contains two Scharlemann cycles with distinct label pairs, contradicting Proposition 2.4.

Proposition 4.4. If $\Gamma_{2}$ does not have property $(*)$, then $\triangle\left(r_{1}, r_{2}\right) \leq 2$.

Proof. Suppose, otherwise, that $\triangle\left(r_{1}, r_{2}\right) \geq 3$. By Proposition 3.5, there exists a vertex of $\Gamma_{1}$, say $x$, such that $\Gamma_{2}^{x}$ contains $2 n_{2}+l$ edges, where $l \geq 1$. By the Euler characteristic formula, $\Gamma_{2}^{x}$ contains at least $n_{2}+l$ disk faces. By the parity rule and Proposition 3.5, there are $n_{2}-l$ vertices of $\Gamma_{2}$, each of which is incident to an edge connecting it to an antiparallel vertex. That means that there are at least $n_{2}-l$ edges of $\Gamma_{2}^{x}$, each of which is on the boundary of only one disk face of $\Gamma_{2}^{x}$. We claim that $\Gamma_{2}^{x}$ contains either a 2 -sided disk face, or a 3 -sided disk face.

If $\Gamma_{2}^{x}$ contains no 2 -sided disk face or 3 -sided disk face. Then $4 F \leq$ $2\left(2 n_{2}+l\right)-\left(n_{2}-l\right)$. Thus $F \leq 3 / 4\left(n_{2}+l\right)<n_{2}+l$, a contradiction.

Now by Lemma 2.6(1), $\Gamma_{2}$ contains a Scharlemann cycle. By Lemma 2.2, $F_{1}$ is separating. By Lemma 2.6(2) and Proposition 2.4, $\Gamma_{2}$ contains at least $n_{2}+l$ Scharlemann cycles with the same label pair, say $\{1,2\}$. Now suppose that $\Gamma_{2}$ contains $m$ Scharlemann cycles with label pair $\{1,2\}$. Then $m \geq n_{2}+l$, where $l \geq 1$. By Lemma 2.5(5), $\Gamma_{2}^{1}$ contains at least $2 m$ edges. By the Euler characteristic formula, $\Gamma_{2}^{1}$ contains at least $m+l$ disk faces. By Lemma 2.6(2), $\Gamma_{2}$ contains at least $m+l$ Scharlemann cycles. Thus $\Gamma_{2}$ contains two Scharlemann cycles with distinct label pairs, contradicting Proposition 2.4.

Theorem 1 follows immediately from Propositions 4.1-4.4.

I am grateful to the referee for his suggestions.

\section{References}

[1] S. Boyer and X. Zhang, Reducing Dehn filling and toroidal Dehn filling, Topology and Its Appl., 68 (1996), 285-303.

[2] M. Culler, C. Gordon, J. Luecke and P. Shalen, Dehn surgery on knots, Ann. of Math., 125 (1987), 237-300.

[3] C. Gordon, Boundary slopes of punctured tori in 3-manifolds, preprint.

[4] C. Gordon and R. Litherland, Incompressible planar surfaces in 3-manifolds, Topology and Its Appl., 18 (1984), 121-144.

[5] C. Gordon and J. Luecke, Reducible manifolds and Dehn surgery, Topology, 35 (1996), 385-403.

[6] C. Hayashi and K. Motegi, Dehn surgery on knots in solid tori creating essential annuli, Trans. AMS, to appear.

[7] M. Scharlemann, Producing reducible 3-manifolds by surgery on a knot, Topology, 29 (1990), 481-500. 
[8] Y-Q Wu, Incompressibility of surfaces in surgered 3-manifold, Topology, 31 (1992), 271-279.

[9] Y-Q Wu, The reducibility of surgered 3-manifolds, Topology and its Appl., 43 (1992), 213-218.

Received December 8, 1996 and revised September 10, 1998. This research was supported in part by National Natural Science Foundation of China.

Department of Mathematics

JiLIN UNIVERSITY

ChangChun 130023

CHina

E-mail address: qrf@mail.jlu.edu.cn 